\title{
COPP-ABV Regimen
}

National Cancer Institute

\section{Source}

National Cancer Institute. COPP-ABV Regimen. NCI Thesaurus. Code C67174.

A regimen consisting of cyclophosphamide, vincristine, prednisone and procarbazine (COPP) alternating with doxorubicin, bleomycin and vinblastine (ABV), used in combination with radiation therapy for the treatment of low-risk, childhood Hodgkin lymphoma. 